\title{
Contact Reflectance Spectroscopy for Rapid, Accurate, and Nondestructive Phytophthora infestans Clonal Lineage Discrimination
}

\author{
Kaitlin M. Gold, ${ }^{1, \dagger}$ Philip A. Townsend,2 Eric R. Larson, ${ }^{1}$ Ittai Herrmann, ${ }^{3}$ and Amanda J. Gevens ${ }^{1}$ \\ ${ }^{1}$ Department of Plant Pathology, University of Wisconsin-Madison, 1630 Linden Drive, Madison, WI 53706, U.S.A. \\ 2 Department of Forest and Wildlife Ecology, University of Wisconsin-Madison, 1630 Linden Drive, Madison, WI 53706, U.S.A. \\ ${ }^{3}$ The Robert H. Smith Institute for Plant Sciences and Genetics in Agriculture, The Hebrew University of Jerusalem, P.O. Box 12, Rehovot \\ 7610001, Israel \\ Accepted for publication 23 December 2019.
}

\begin{abstract}
Populations of Phytophthora infestans, the oomycete causal agent of potato late blight in the United States, are predominantly asexual, and isolates are characterized by clonal lineage or asexual descendants of a single genotype. Current tools for clonal lineage identification are time consuming and require laboratory equipment. We previously found that foliar spectroscopy can be used for high-accuracy pre- and postsymptomatic detection of $P$. infestans infections caused by clonal lineages US-08 and US-23. In this work, we found subtle but distinct differences in spectral responses of potato foliage infected by these clonal lineages in both growth-chamber time-course experiments (12to 24-h intervals over 5 days) and naturally infected samples from commercial production fields. In both settings, we measured continuous visible to shortwave infrared reflectance (400 to $2,500 \mathrm{~nm}$ ) on leaves using a portable spectrometer with contact probe. We consistently discriminated between infections caused by the two clonal
\end{abstract}

ABSTRACT lineages across all stages of disease progression using partial least squares (PLS) discriminant analysis, with total accuracies ranging from 88 to $98 \%$. Three-class random forest differentiation between control, US-08, and US-23 yielded total discrimination accuracy ranging from 68 to $76 \%$. Differences were greatest during presymptomatic infection stages and progressed toward uniformity as symptoms advanced. Using PLS-regression trait models, we found that total phenolics, sugar, and leaf mass per area were different between lineages. Shortwave infrared wavelengths $(>1,100 \mathrm{~nm})$ were important for clonal lineage differentiation. This work provides a foundation for future use of hyperspectral sensing as a nondestructive tool for pathovar differentiation.

Keywords: analytical and theoretical plant pathology, ecology and epidemiology, techniques,
Late blight of potato and tomato, caused by the oomycete plant pathogen Phytophthora infestans, is one of the most challenging diseases to sustainably and proactively manage. P. infestans is a heterothallic pathogen with two mating types, A1 and A2 (Fry 2008). In cooccurrence, A1 and A2 mating types can reproduce sexually, producing oospores capable of survival for long periods of time. Sexual recombination can result in increased genetic diversity, yielding increased virulence, altered host range, or fungicide resistance. In the United States, however, populations of the pathogen are predominantly asexual and isolates are characterized by clonal lineage. This is determined by mitochondrial haplotype, nuclear DNA fingerprint pattern, allozyme genotype, and mating type (Fry 2008; Goodwin et al. 1995). A clonal lineage is defined as the asexual descendants of a single genotype (Anderson and Kohn 1995).

†Corresponding author: Kaitlin M. Gold; kmorey@wisc.edu

Funding: Support was provided by graduate research funding from the Accelerator Program administered through the Wisconsin Alumni Research Foundation, the Wisconsin Specialty Crop Block Grant Program administered through the Wisconsin Department of Trade and Consumer Protection, and the University of Wisconsin Madison College of Agricultural and Life Sciences for Potato Industry Board fellowship funds awarded competitively to K. Gold; and by Wisconsin potato growers, who provided support and partnership in conducting research and providing extremely useful feedback. Additional support was provided to P. A. Townsend by the United States Department of Agriculture Hatch grant WIS01874 and NASA Advanced Information Systems Technology (AIST) grant 80NSSC17K0244.

*The $\boldsymbol{e}$-Xtra logo stands for "electronic extra" and indicates that four supplementary figures and three supplementary tables are published online.

The author(s) declare no conflict of interest.

(c) 2020 The American Phytopathological Society
Although all individuals of a clonal lineage are descended from a single genotype, there can be diversity within that population of individuals (Fry and Goodwin 1997; Seidl and Gevens 2013; Seidl Johnson and Gevens 2014; Seidl Johnson et al. 2015a).

Clonal lineage US-08 was one of the first A2 mating types found in the United States and is notorious for its association with P. infestans epidemics of the late 1980s and 1990s. Within 5 years of its first detection in 1992, US-08 had spread to three-fifths of the continental United States (Fry and Goodwin 1997). Until 1984, A1 mating types dominated $P$. infestans populations outside of Mexico. US-08 is resistant to mefenoxam, a highly effective phenylamide fungicide commonly relied upon for oomycete disease management (Deahl et al. 1991, 1993; Fry and Goodwin 1997). At the time of these epidemics, mefenoxam (the more active enantiomer of metalaxyl) was the only systemic late blight fungicide available globally.

In 2009, a pandemic caused by newly emerged $P$. infestans clonal lineages destroyed commercial, small-acreage farm, and home garden tomato and potato crops across the United States (Fry et al. 2013, 2015; Seidl and Gevens 2013). The 2009 pandemic was caused by the mass distribution of clonal lineage US-22 via infected tomato transplants from a national supplier sold in large retail stores across the United States (Fry et al. 2013). In subsequent years, the mefenoxam-sensitive clonal lineage US-23 emerged and became an annual occurrence in humid potato-growing regions of the United States. Like US-08, US-23 is aggressive on both potato and tomato (Fry et al. 2015; Hansen et al. 2014). US-23 has demonstrated greater fitness than other contemporary clonal lineages, with increased sporangial production, smaller sporangial size, and increased tolerance to freezing temperatures (Frost et al. 2015; Seidl Johnson et al. 2015a). Clonal lineages can vary not only in fungicide resistance but also in host range (Seidl Johnson et al. 2014, 2015a), sensitivity to temperature (Frost et al. 2015; Seidl Johnson et al. 2015b), and general 
aggressiveness (Pariaud et al. 2009). Plant response to infection from different clonal lineages can also result in variation in defense gene activation and suppression (Wang et al. 2008) and in the leaf metabolome (Henriquez et al. 2012).

Clonal lineage identification has been important for practical late blight management since the emergence of mefenoxam-resistant isolates in the 1990s. Unlike US-08 isolates, most US-23 isolates are sensitive to mefenoxam (Seidl and Gevens 2013). The ability to rapidly determine clonal lineage identity is important for fungicide selection and management decision-making. Current identification methods such as serological or molecular testing are time consuming and are not easily or rapidly deployed at large scales (Seidl and Gevens 2013). The glucose-6-phosphate isomerase allozyme assay has been the most widely replied-upon identification method for over 20 years (Goodwin et al. 1995). This assay can identify clonal lineage and associated fungicide resistance within less than $24 \mathrm{~h}$ of sample receipt; however, is only effective when sporangia are present. Before this method can be used, a new clonal lineage must be identified by molecular biological means and an allozyme-banding profile associated (Danies et al. 2013; Small et al. 2012). The ability to render a preliminary determination of the clonal lineage identity on site would facilitate faster prescriptive management.

Reflectance spectroscopy has emerged as a powerful and promising tool for crop disease detection in a variety of pathosystems (Alisaac et al. 2018; Baranowski et al. 2015; Bergsträsser et al. 2015; Couture et al. 2018; Heim et al. 2018; Herrmann et al. 2018; Kuska et al. 2015; Liu et al. 2010; Mahlein et al. 2010, 2012, 2013; Rumpf et al. 2010; Thomas et al. 2018; Wahabzada et al. 2015; Zarco-Tejada et al. 2018). Despite this wealth of literature, the potential use of field-based spectroscopy to rapidly and nondestructively estimate plant responses to pathogen infection has not been implemented in research or commercial field settings. To date, a number of foliar biochemical, physiological, structural, and morphological properties have been successfully quantified using reflectance spectroscopy (Asner and Martin 2008, 2011; Couture et al. 2013, 2016; Curran 1989; Gillon et al. 1999; Kokaly et al. 2009; Kokaly and Skidmore 2015; Petisco et al. 2006; Yuan et al. 2016), yet these discoveries have not been widely applied in basic phytopathological studies.

The use of spectroscopy to differentiate between the biochemically and physiologically diverse spectral responses to pathogen species and strain has had limited exploration. Unique variants within a pathogen species may yield different physiological and biochemical responses with potential for spectral differentiation. Hyperspectral reflectance and thermal imaging have been used to detect and differentiate among four closely related Alternaria spp. with $80.5 \%$ accuracy (Baranowski et al. 2015). Arens et al. (2016) linked their capacity for presymptomatic detection to differences in various metabolite concentrations across resistant and susceptible cultivars. Couture et al. (2018) examined four potato virus Y strains and found they produced different spectral profiles but were only able to achieve $12 \%$ discrimination accuracy using partial least squares discriminant analysis (PLS-DA). Alisaac et al. (2018) performed multiclass differentiation using support vector machines between a control and two closely related species of Fusarium on wheat (Fusarium graminearum and F. culmorum) with $76 \%$ accuracy.

The ability to nondestructively identify $P$. infestans clonal lineage would greatly reduce the lag time between pathogen detection and management response. Furthermore, identification of unique host responses to clonal lineages opens up a new area of basic host-pathogen research that could enhance our understanding of resistance and pathogen virulence. Previously, we discovered that there are sufficient commonalities among infections caused by specific $P$. infestans clonal lineages to allow for highly accurate detection at all disease time stages (Gold et al. 2019). The goal of the present work was to identify where there are no commonalities between US-08 and US-23 to better understand how spectroscopic methods can be used to differentiate between visually identical infections, and to characterize the underlying differences in disease physiology that form the basis for this capacity. This work forms the foundation for future nondestructive and rapid diagnosis of clonal lineages with spectroscopic systems. The specific objectives of this work were to (i) evaluate the differences in spectral reflectance between potato leaves infected with clonal lineages US-08 and US23 of $P$. infestans and (ii) quantify the effects of infection with US08 and US-23 on spectrally derived biochemical and physiological trait indices.

\section{MATERIALS AND METHODS}

Plant and pathogen materials. Katahdin potato plants were grown for 4 weeks from axenic tissue culture cuttings in pathogenfree growth chambers with a 12 -h photoperiod under $24^{\circ} \mathrm{C}$ daytime temperature and $21^{\circ} \mathrm{C}$ nighttime temperatures. Axenic, hyphal-tipderived $P$. infestans isolates of clonal lineages US-08 and US-23 were obtained from naturally infected commercial potato fields in 2017 from central Wisconsin. These samples were isolated, cleaned, and placed into long-term storage on sterilized hemp seed (Lamour and Hausbeck 2002). This was done to avoid culturing the isolate more than twice prior to use in experimentation (Fry et al. 2019). To obtain working cultures, colonized hemp seed were placed onto rye A agar plates and grown for 3 weeks at $18^{\circ} \mathrm{C}$ in darkness. Subsequently, all cultures were placed at room temperature under ambient lighting for 24 to $48 \mathrm{~h}$ to induce sporangial production prior to inoculation. A 5-mm agar plug was excised from cultures in areas with concentrated sporulation and inoculated, sporangia side down, onto the adaxial surface of the leaf. After inoculation, plants were placed into humidity chambers to ensure $100 \%$ relative humidity to support infection. Treatments were maintained in separate humidity chambers to prevent crosscontamination and reduce the likelihood of inoculum spread during measurement. Treatments were assigned randomly to humidity chambers and varied across the three independent experiments. Treatments included rye A agar plug mock inoculation (control), US-08 inoculation, US-23 inoculation, and a combined US-08 and US-23 mixed inoculation. Twenty replicates were used per treatment and two leaves were measured per plant. Disease was rated on a scale from 0 to 5 , with $0=$ no disease and $5=$ severe disease at each time point using a Horsfall-Barratt scale (Barratt and Horsfall 1945). Our scale was modified so that 0 indicated no disease and 1 indicated nonnecrosis but with mild cellular collapse when examined microscopically (Fig. 1Aii). At no time point did disease surpass a Horsfall-Barratt rating of 5 (12 to $25 \%$ of leaf visibly diseased). Using the disease ratings, area under the disease progress curve (AUDPC) was calculated over the study period (up to $120 \mathrm{~h}$ postinoculation) to determine whether infection progressed similarly for the two clonal lineages in our study. Infection was confirmed with an enzyme-linked immunosorbent assay immunostrip assay for the genus Phytophthora (Agdia). Samples that did not achieve a disease rating of $\geq 4$ by the end of the study period were not included in the analysis.

RNA isolation, cDNA synthesis, and quantitative realtime PCR. Quantitative real-time PCR (qRT-PCR) was performed to quantify two important RXLR effectors, Avr3a and SFI3, known to be differentially expressed by US-08 and US-23 during early infection and biotrophy (Armstrong et al. 2005; Zheng et al. 2014) to provide a biological basis for the differences between the clonal lineages and relate differential disease physiology to inferences derived from spectral reflectance. Fifteen Katahdin potato plants were grown and inoculated following the conditions and treatments previously described. One leaf was destructively sampled at 24-h intervals starting at $24 \mathrm{~h}$ postinoculation (hpi) and ending at $96 \mathrm{hpi}$. Infection did not progress to sporulation during this study period. A 2.5-cm square at the point of inoculation (but not including the inoculation plug) was excised from each leaf and used for RNA 
extraction. Total RNA was extracted by bead-beating in conjunction with the Maxwell RSC Plant RNA Kit and Maxwell RSC Instrument (Promega Corp.). cDNA was synthesized using GoScript Reverse Transcription Mix with random primers (Promega Corp.). qRT-PCR reactions were performed in triplicate with GoTaq qPCR Master Mix (Promega Corp.) and run on a Bio-Rad C1000 Touch Thermal Cycler with a CFX96 Real-Time System. Relative expression was calculated using CFX Maestro Software (Bio-Rad). The relative expression of $A v r 3 a$ and $S F I 3$ was determined using ActA as a reference gene. Primers and reaction protocols can be found in Supplementary Table S1.

Reflectance measurements. Leaf reflectance spanning the visible (VIS) to shortwave infrared (SWIR) was measured using two high-spectral-resolution SVC HR-1024i (350 to 2,500 nm; collectively VSWIR) portable spectroradiometers (Spectra Vista Corporation), whereby each spectrometer measured a portion of each treatment. Spectrometer effect was assessed using permutation multivariate analysis of variance (PERMANOVA) and no significant difference between instruments was found $(P>0.05)$. All measurements were taken from the leaf adaxial surface using a leafclip assembly attached to a plant probe with an internal halogen light source, using $99 \%$ spectralon as white reference (Labsphere). Reflectance was measured on two locally inoculated leaves per plant with two spectra averaged per leaf location to determine the mean leaf reflectance. Measurements were taken immediately above the inoculation zone but not on top of the inoculation plug. Baseline measurements were taken immediately prior to inoculation and then at 12- to 24-h intervals for the next 5 days. Bad measurements such as those with low reflectance or abnormalities due to measurement error were removed as well, yielding 333 individual samples and 1,559 total spectral measurements over time. Pictures of leaves were taken at each time point to document phenotypic changes (Nikon D200; Nikon USA Inc.).
Field sample collection. Asymptomatic and symptomatic (with sporulation) leaves were opportunistically measured in commercial production fields in central Wisconsin in August 2017 and August 2018. Samples from August 2017 were of potato cultivar Russet Norkotah infected by P. infestans clonal lineage US-08. Samples from August 2018 were of potato cultivar Russet Ranger infected by $P$. infestans clonal lineage US-23. Clonal lineage identity was determined by allozyme analysis (Goodwin et al. 1995). All spectral measurements were taken from the leaf adaxial surface on green tissue directly adjacent to necrotic lesions using a leaf-clip assembly attached to a plant probe with a halogen light source. Two spectra were averaged per leaf to determine the mean leaf reflectance. Leaves were categorized as "diseased" if they had late blight lesions (2017: $n=217$ and 2018: $n=88$ ) and "nondiseased" if they had no lesions or other apparent symptoms of late blight (2017: $n=517$ and 2018: $n=95)$. Leaves were removed, placed into sealed plastic bags with wet paper towels to encourage disease development, and brought back to the lab to be monitored for late blight symptom development over the course of $48 \mathrm{~h}$. It could not be determined whether nondiseased leaves that developed symptoms over the course of monitoring had been infected in the field or during the handling process, so if nondiseased leaves developed symptoms, they were removed from the analysis.

Data preparation. For analysis, we utilized reflectance measurements from 400 to $2.400 \mathrm{~nm}$, removing noisy bands at the shortest and longest wavelengths. Data were interpolated to $1-\mathrm{nm}$ resolution from the native 3- to $8-\mathrm{nm}$ resolution of the instrument. Bad measurements such as those with low reflectance or other abnormalities due to measurement error were removed prior to data analysis (Couture et al. 2018; Serbin 2012; Singh et al. 2015; Zhai et al. 2013).

The stage of disease development (Gold et al. 2019) was defined for statistical analyses as follows: inoculated samples were labeled

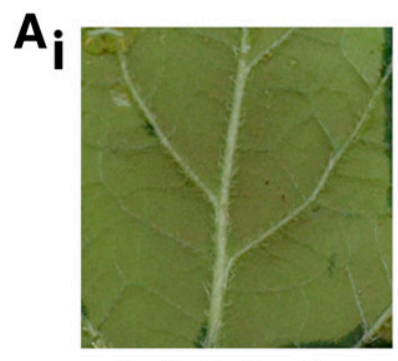

Early Infection

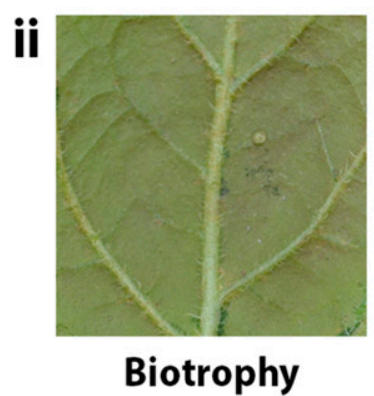

Biotrophy

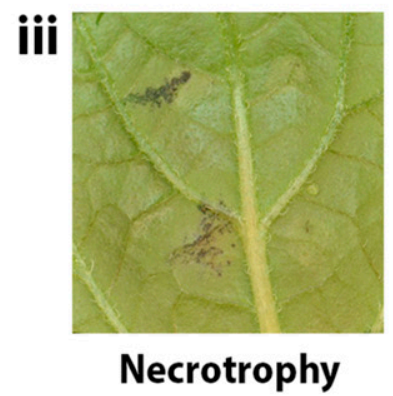

C

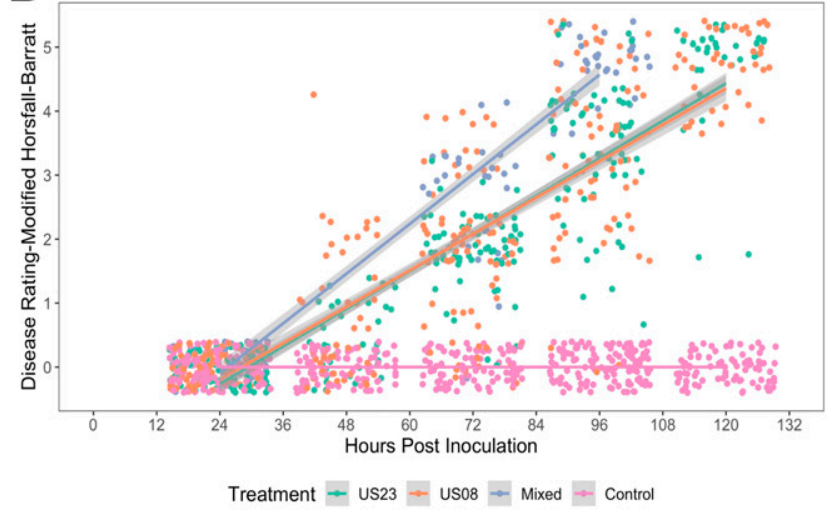

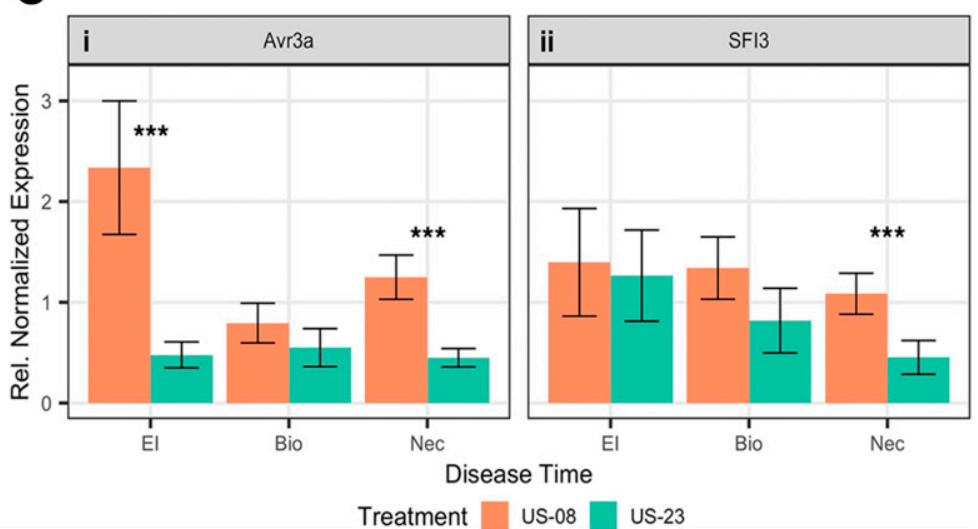

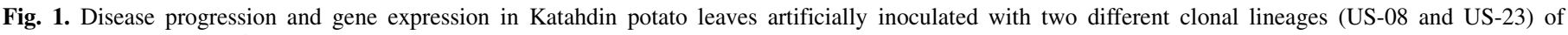

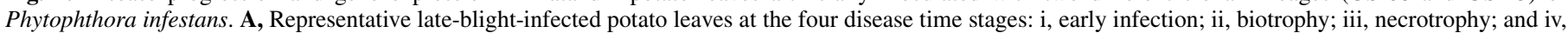

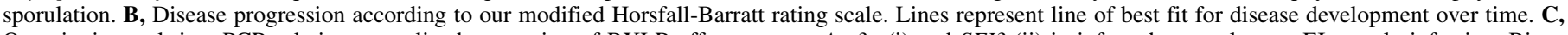

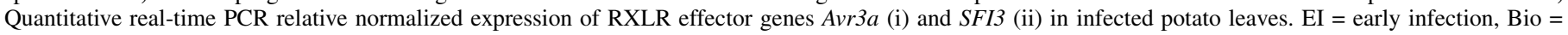

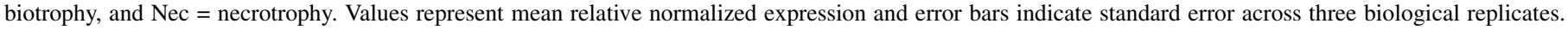
Asterisks $(* * *)$ indicate $P<0.001$. 
"early infection" at time points within $24 \mathrm{~h}$ of inoculation, "biotrophy" during measurement time points spanning $48 \mathrm{~h}$ until the last time point made before disease symptoms appeared, "necrotrophy" during time points after which disease symptoms had become visible but before sporulation occurred, and "sporulation" when both necrosis and sporulation were visible. Disease development stage was defined for control plants as the approximate range of time during which the majority of inoculated plants entered into the next infection progression stage. This was done so that the interaction of treatment, clonal lineage, and time could be estimated while accounting for the fact that not all inoculated individuals progressed uniformly in infection development and disease severity, and so that spectral changes caused by plant response to measurement and handling could be differentiated from those caused by infection.

Data analysis. All data analyses were conducted in $\mathrm{R}$. PERMANOVA was conducted to generate "pseudo" $F$ and $P$ values to test significance of treatment on spectral reflectance using the R package vegan (Oksanen et al. 2013). The PERMANOVA randomization was restricted (blocked) by experiment and stratified by sample ID and disease development stage to account for the repeated-measures nature of the experiment (Anderson and Walsh 2013). Principal coordinates analysis (PCoA) was used to visualize broad spectral differences between infection caused by the two clonal lineages using spectral reflectance as the predictor variable with the $\mathrm{R}$ package vegan (Oksanen et al. 2013). This method uses a dissimilarity matrix calculated using Euclidean distance to transform a large number of possibly correlated predictor variables into a smaller number of uncorrelated latent variables, reducing the dimensionality of the data.

Normalized difference spectral indices (NDSI) were calculated for all possible combinations of wavelengths to identify regions of the spectrum most correlated (Pearson correlation) with change in infection status. Clonal lineage infection status was correlated separately for US-08 and US-23 with each normalized index (approximately 4 million combinations) using Pearson's correlation and plotted in a wavelength-versus-wavelength NDSI heat map to illustrate the areas of the spectrum most different between the two lineages, which can be interpreted as the identification of narrowband spectral indices that best discriminate infections. NDSI graphs through time help visualize how the spectra of infected plants change over the course of disease development between the two isolates. The top $0.1 \%$ of most correlated NDSIs were then tabulated to identify the wavelengths most frequently associated with discrimination between treatments. The relative frequency of a wavelength by clonal lineage and disease developmental stage was plotted to visualize how the progression of disease is expressed spectrally.

Foliar nitrogen, total phenolics, sugar, starch, and leaf mass/area (LMA) were estimated from spectra using partial least squares regression (PLSR) calibrations from Chlus et al. (unpublished) posted under UW-ENSPEC profile on EcoSML.org, an open-source repository of spectral mapping functions. Because these models were not specifically validated in our study, they must be considered to be trait indices rather than true trait quantities; however, they yield important insights into the underlying physiological and biochemical drivers of spectral response nonetheless. LMA is an indicator of leaf dry-mass investment and relates to light interception and leaf longevity (Reich 2014; Wright et al. 2004). The normalized difference water index (NDWI) was calculated using the relative difference in reflectance at 857 and $1,241 \mathrm{~nm}$ as an indicator of leaf water content (Gao 1996). A two-way, repeated-measures analysis of variance (ANOVA) was fit using a mixed-effects model with maximum-likelihood estimation method for each of the metrics total phenolics concentration, sugar concentration, starch concentration, nitrogen concentration, NDWI, and LMA using the R package nmle (Pinheiro et al. 2013). The R package emmeans was used to understand interaction effects for treatment across time (Lenth 2018). Treatment was fit as a fixed effect, with sample identifier and disease development stage modeled as random effects to account for the repeated-measures nature of the experiment. To preserve balance in the model, mixed infection samples were not included in this analysis due to low sample size.

PLS-DA was used to determine how well clonal lineage could be classified with the R packages caret and pls (Kuhn 2015; Mevik et al. 2011). PLS-DA is widely used to discriminate groups with independent variables having high dimensionality and collinearity. PLS-DA projects latent vectors (or components) through the response and predictor variables to both reduce data dimensionality and maximize prediction accuracy (Worley and Powers 2013). PLS approaches do not directly provide parameter uncertainties; therefore, the analyses were permutated (100 times) by dividing observations by groups (70:30) into training (calibration) and testing (cross-validation) sets to estimate jack-knifed uncertainty. The number of correct classifications in the validation sets across the permutations and $\kappa$ statistic was used to evaluate the tested models (classification accuracy). Internal cross-validation is essential due to the tendency of PLS approaches to overfit (Wold et al. 2001; Worley and Powers 2013). The ideal number of latent vectors was identified by iteratively running the PLS-DA models with increasing numbers of components and examining the predicted residual error sum of squares (PRESS), selecting the number of latent vectors that minimized PRESS across all permutations. The absolute values of the averaged standardized coefficients across 100 simulations were extracted and ranked to determine important regions of the spectra for classification. PLS-DA models were fit for differentiating between US-08-infected leaves and US-23-infected leaves across all time points as well as at each disease time stage.

Random forest (RF) classification, an ensemble machinelearning approach, was employed to assess three-class discrimination accuracy (control, US-08, and US-23) and to compare with PLS-DA performance using the R package caret (Kuhn 2015). Multiclass differentiation with PLS-DA takes a one-versus-all approach, which is why we chose to employ a different method for comparison. RF classification is a nonparametric method that uses multiple decision trees to classify data well suited to spectral data analysis (Breiman 2001; Hastie et al. 2009; Heim et al. 2018; Immitzer et al. 2012). For RF classification, the dataset was resampled to $10-\mathrm{nm}$ resolution (202 wavebands) with the prospectr package in R (Stevens and Ramirez-Lopez 2014) to yield an optimal number of predictor variables and to reduce collinearity among wavelengths. RF was applied separately to both primary and firstorder derivative (FOD) spectra to enhance spectral features (Demetriades-Shah et al. 1990). The RF model was optimized in an 80:20 training/testing data subset to establish the two parameters primarily responsible for model performance-the number of randomly selected predictors to choose from at each split (mtry) and number of trees generated to yield a full ensemble ( $n$-tree) - and 10fold repeated internal cross-validation was used to calculate out-ofbag, or bootstrapped prediction, error. This process was repeated 10 times and the mean accuracy and $\kappa($ Cohen 1960) were calculated to gauge model performance. The $\kappa$ values can be interpreted using standards established by Landis and Koch (1977): $0=$ poor, 0.1 to $0.2=$ slight, 0.2 to $0.4=$ fair, 0.4 to $0.6=$ moderate, 0.6 to $0.8=$ substantial, and 0.8 to $1=$ near perfect. Final model parameters chosen were $n$-tree $=500$ with variable $m$ try, number of branches per tree per model fit. Average test accuracy $\kappa$, overall accuracy, false-negative rate, and false-positive rate were used to determine model performance.

\section{RESULTS}

Effector expression differs between clonal lineages and may contribute to broad spectral differences between clonal lineages. During early infection and biotrophy, no visible symptoms of necrosis were present (Fig. 1A). Disease progressed similarly for all treatments included in this study (Fig. 1B). There was no significant difference between the AUDPC between mixed-, 
$P$. infestans US-08-, and $P$. infestans US-23-inoculated samples but all were significantly different from the control (ANOVA, Tukey's post-hoc test, $P<0.05$ ).

During early infection, Avr3a relative expression was significantly higher in US-08- than US-23-infected leaves (Fig. 1C). As the infection progressed to biotrophy ( $48 \mathrm{hpi}$ ), a stage defined by the appearance of minor water-soaking symptoms but without necrosis, Avr $3 a$ relative expression became more similar between the clonal lineages. Between 72 and 96 hpi, US-08 and US-23 infections progressed to necrotrophy and US-08 Avr $3 a$ expression was found to be significantly higher than that of US-23. Over the course of infection, Avr3a expression was relatively consistent in US-23infected samples, whereas expression fluctuated in US-08-infected samples, peaking significantly during early infection. SFI3 expression was more consistent across all stages of infection for both clonal lineages, decreasing slightly as disease progressed. SFI3 expression was significantly different between clonal lineages during necrotrophy (Fig. 1C).

Overall, there was considerable similarity between the spectra of foliage infected by the two clonal lineages. Visible examination of spectra showed little or no discernable differences among leaves of US-08, US-23, or mixed infection (Supplementary Fig. S1). PCoA confirmed this observation, showing that spectra from US-08, US-23, and mixed-infection samples exhibited separation from control samples only when pooling all disease development stages (Fig. 2). The inoculated samples showed greater separation from each other during early infection than during sporulation (Fig. 2). This suggests differences in the underlying physiological response to clonal lineage infection. Together, the first two PCoA components explained $95 \%$ of the variation seen within spectra. PERMANOVA showed a significant difference between US-08 and US-23 spectra (pseudo $P=0.001$ ) and that disease development stage did not significantly affect spectral reflectance (pseudo $P=0.1618$ ). The interaction of treatment and disease stage was not significant (pseudo $P=0.3896$ ), indicating that differences between spectra of infected leaves were consistent regardless of disease progression (Supplementary Table S2).

Spectrally derived leaf foliar traits illustrate underlying similarities and differences between clonal lineage disease physiology. Leaf biochemical concentrations and morphology were estimated with PLSR trait models from Chlus and Townsend (unpublished data) and posted on EcoSML.org. These trait indices identified differences in how clonal lineage affects overall leaf health (Supplementary Table S3; Fig. 3). Spectrally derived traits were different

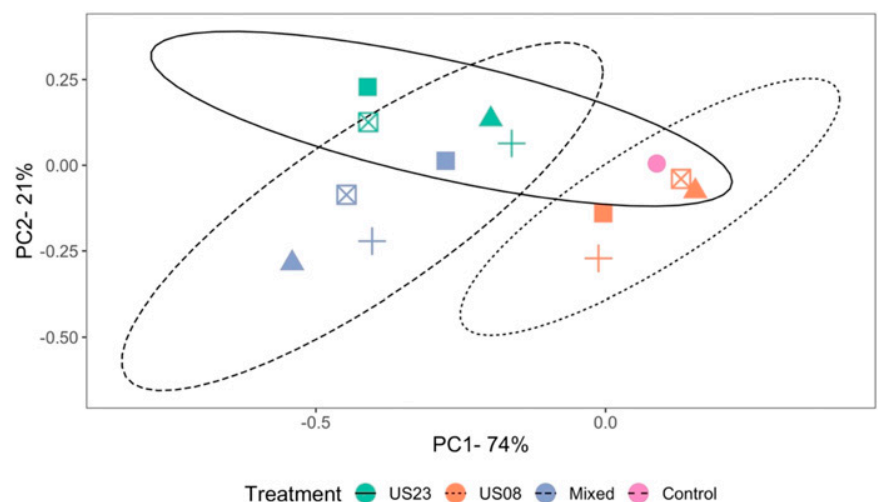

Disease Time $\boldsymbol{Q}$ Control $\boldsymbol{\Delta}$ Early Infection $\boldsymbol{\square}$ Biotrophy + Necrotrophy $\boldsymbol{\otimes}$ Sporulation

Fig. 2. Principal coordinate (PC) analysis plots illustrating spectral separation between potato plant responses to infection by Phytophthora infestans clonal lineages US-08 and US-23 in a growth chamber, calculated from visible to shortwave infrared reflectance (400 to $2,500 \mathrm{~nm}$ ) on leaves using a portable spectrometer with contact probe at $12-$ to $24-\mathrm{h}$ intervals for 5 days. Points represent average value for leaves of each class and disease time combination. Ellipses represent $95 \%$ confidence interval. between the lineages and mixed infections when compared with control and across disease development. Disease stage, treatment (US08, US-23, and control), and the interaction term were significant in every model (Supplementary Table S3). Total phenolics concentration and LMA were significantly different between US-08 and US23 at all disease time points (Fig. 3A and F). US-08-infected leaves consistently had greater phenolics concentrations than control and US-23-infected leaves (Fig. 3A). US-23-infected leaves had greater LMA values than both control and US-08-infected leaves at all disease time points (Fig. 3F). Leaf sugar concentration was significantly different between US-08- and US-23-infected leaves only during presymptomatic stages (Fig. 3B). Mixed-infection leaves were significantly different from control leaves for five of six (excluding NDWI) spectrally derived traits at all stages, except for sugar concentration during sporulation (Supplementary Table S3). Comparisons were not made for the mixed-infection treatment during biotrophy due to low sample number $(n=3)$.

Measurements were made on infected leaves in commercial production fields in August 2017 (US-08, Russet Norkotah) and August 2018 (US-23, Russet Ranger). These samples could not be directly compared due to the confounding effect of cultivar but useful observations were made nonetheless. Physiological and biochemical trait indices were fit and the percent relative difference from nondiseased leaves compared between the two clonal lineages (Fig. 4). Both US-08 and US-23 infections in commercial samples caused an increase in total phenolics and nitrogen concentration and a decrease in foliar sugar and starch concentrations, LMA, and NDWI relative to their respective control plant samples (Fig. 4). When the diseased and nondiseased leaves were directly compared for each collection year, we saw that all five traits were significantly different between US-08-affected and unaffected Russet Norkotah leaves (Supplementary Fig. S2). All traits were significantly different between US-23-infected and noninfected leaves except for total phenolics concentration and NDWI (Supplementary Fig. S2).
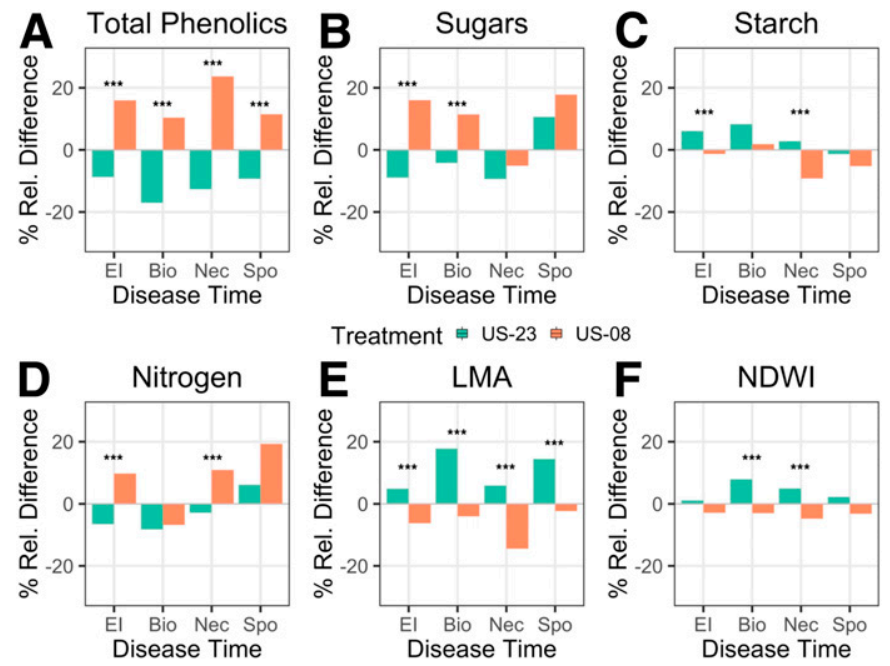

Fig. 3. Percent relative difference (calculated from group averages) between spectrally derived physiological and biochemical leaf trait indices for Katahdin potato leaves inoculated with Phytophthora infestans clonal lineages US-08 and US-23 and control, noninoculated leaves. Trait indices derived from partial least squares regression models built for agricultural crops and calculated using visible to shortwave infrared reflectance (400 to $2,500 \mathrm{~nm}$ ) on leaves using a portable spectrometer with contact probe at 12- to 24-h intervals for 5 days. EI = early infection, Bio = biotrophy, Nec = necrotrophy, and Spo = sporulation. A, Total phenolics; B, sugars; C, starch; D, nitrogen; E, leaf mass per area (LMA); and F, normalized difference water index (NDWI). Asterisks (***) indicate $P$ value $<0.001$ for pairwise comparison of difference between raw concentration values. Raw concentration values are illustrated in Supplementary Figure S2. 
Comparison of symptomatic Russet Ranger leaves infected with US-23 and Russet Norkotah leaves infected with US-08 in commercial fields allowed us to better understand differences between spectral profiles of different lineages in potato of a similar parentage at more advanced stages of infection (sporulation) (Supplementary Fig. S3). Both lineages caused a similar effect on the measured physiological and biochemical traits, causing an increase in total phenolics and nitrogen concentration; and decreases in sugar and starch concentrations, LMA, and NDWI, providing more support for the conclusion that both spectra and physiological or biochemical response converge at later stages of infection and disease severity.

Multiple discrimination methods identify similar spectral features. NDSI were calculated across all disease time points and within each disease time stage to identify the regions of the spectrum that differed most between clonal lineages (Fig. 5). Overall, the two-band NDSI wavelength combinations that best differentiated the two clonal lineages remained consistent throughout disease development but increased in strength of statistical relationship as infection progressed. For example, during early infection, the interactions of the VIS and SWIR were mildly associated with lineage (Fig. 5B). These regions became more different between lineages during biotrophic and necrotrophic growth, with reduced differentiation during sporulation. Across all time points, NDSIs representing interactions of the VIS and SWIR, within the near infrared (NIR), and within SWIR were the most different between the two lineages (Fig. 5A).

During early infection, narrowband features within the NIR and SWIR differed most between clonal lineages. During biotrophy, the NDSIs most correlated with differences between lineages occurred in the interactions of the VIS through SWIR, NIR, and SWIR (Fig. 5C). During necrotrophy, the interactions of VIS wavelengths became more important. This is not surprising, because necrosis is visible during this stage. During necrotrophy, the interactions of the VIS through SWIR continued to be highly differentiating between lineages, and interactions within the NIR became less intensively different (Fig. 5D). During sporulation, the interactions within the VIS were the most different between the two lineages (Fig. 5E).

These observations were corroborated by examining the distribution of the top $1 \%$ of most correlated NDSIs (Fig. 6). When all time points were considered, the greatest differences between lineages was in VIS wavelengths and within the 1,400- and 1,900$\mathrm{nm}$ water absorption features ("water bands"). The wavelengths associated with the water bands were the most different between US-08 and US-23 at all time points but most intensively so during presymptomatic stages (early infection and biotrophy). The water bands continued to be important as infection progressed to necrotrophy. As infection progressed toward symptom development, the greatest differentiation between lineages continued to be in the VIS wavelengths.

PLS-DA was performed to differentiate between US-08 and US23 infections and identify the most critical spectral regions for discrimination (Table 1). All classification models had internal cross-validation accuracies $>85 \%$ and $\kappa$ values $>0.75$. Across all disease time points, internal cross-validation accuracy was $95 \%$ and $\kappa$ was 0.9 (components $=14)$. The 20 wavelengths with the largest absolute standardized coefficients in the PLS-DA model, representing the top $1 \%$ of coefficients, were in the VIS light region (approximately $500 \mathrm{~nm}$ ) and between 1,870 to $1,890 \mathrm{~nm}$. Lineage differentiation was most accurate during early infection, with a training interval cross-validation accuracy of $98 \%$ and $\kappa=0.97$ (components $=12$ ). During this disease time stage, the wavelengths with the top 20 absolute standardized coefficients were predominantly in the SWIR, with only three wavelengths in the VIS range. The important SWIR wavelengths came from the range of 1,860 to $1,890 \mathrm{~nm}$ and 2,370 to $2,385 \mathrm{~nm}$.

During biotrophy, necrotrophy, and sporulation, cross-validation accuracies ranged from $89 \%$ upward (Table 1). Interestingly, accuracy decreased as symptoms advanced. Internal crossvalidation accuracies were $95.1,90.3$, and $88.5 \%(\kappa=0.90,0.81$, and 0.77 , respectively). During biotrophy, the majority of the wavelengths with the largest absolute standardized coefficients were from 1,860 to $1,890 \mathrm{~nm}$, with three wavelengths from the VIS wavelengths, indicating similarity between the two presymptomatic stages. During necrotrophy and biotrophy the top 20 wavelengths with the highest absolute standardized coefficients in the model had significant overlap and were entirely composed of SWIR wavelengths from the 1,890 s and 2,370 to $2,390 \mathrm{~nm}$. The general overlap in wavelengths with the largest absolute standardized coefficients between the models indicated that spectral differences between US-08 and US-23 were likely the result of a consistent underlying physiological process.

RF classification was fit with both the original spectra and FOD transformed spectra to determine three-class discrimination accuracy between the control and leaves infected by $P$. infestans US-08 and US-23 (Table 2). Using original spectra, the three-class
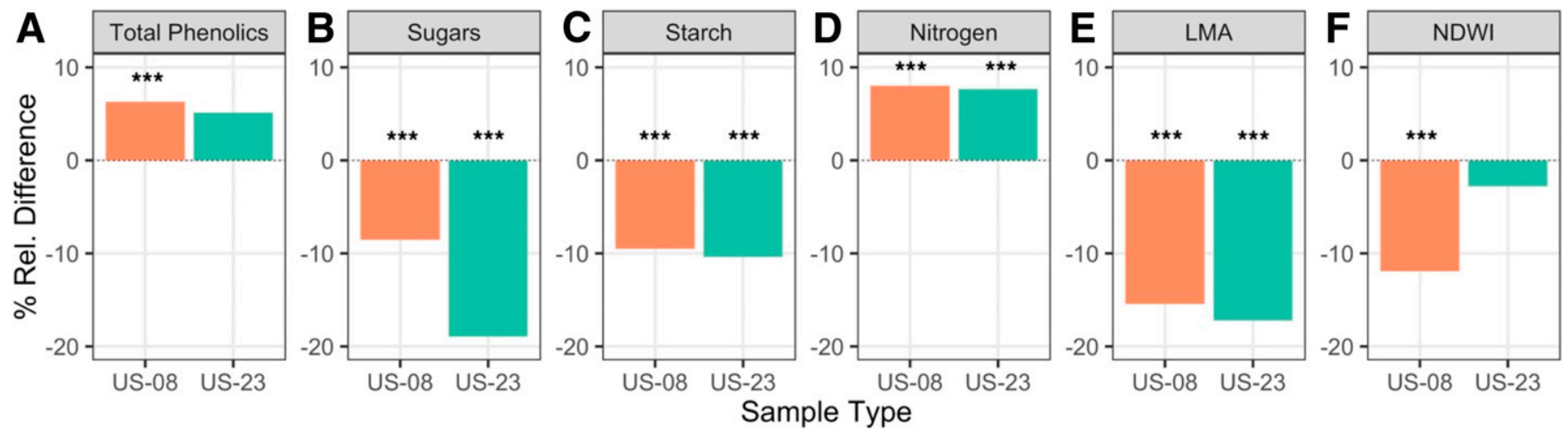

Type $\square$ US-08 $\square$ US-23

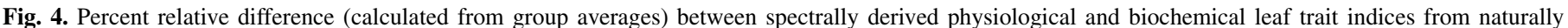

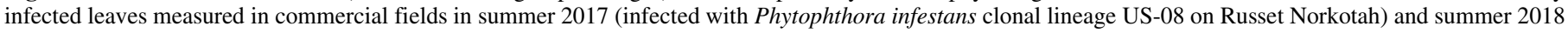

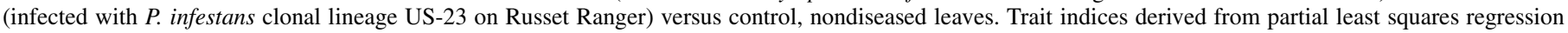

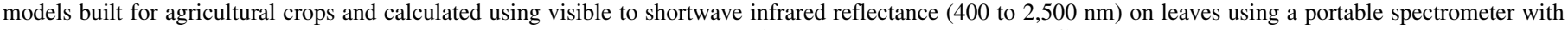

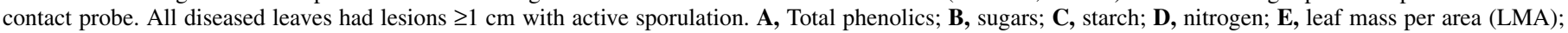

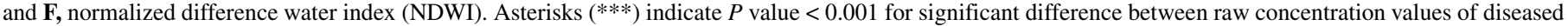
and nondiseased leaves. Raw concentration values are illustrated in Supplementary Figure S3. 
internal cross-validation (out-of-bag) accuracy across all disease time points was $56.9 \%(\kappa=0.29)$, whereas it was $59.8 \%$ across presymptomatic disease time points $(\kappa=0.38)$ and $60.7 \%(\kappa=0.41)$ across postsymptomatic time points. Applying FOD transformation increased model accuracy and $\kappa$. FOD classification accuracy was $69.0 \%(\kappa=0.49)$ across all disease time points, $75.9 \%(\kappa=0.63)$ across presymptomatic disease time points, and $67.9 \%(\kappa=0.43)$ across postsymptomatic disease time points. The top 20 variables important to the projection (VIP) for all models were dominated by SWIR wavelengths but included some VIS wavelengths. Across all disease time points, high VIP values spanned the entire measured spectra and included 5 wavelengths in the VIS range, 5 from the NIR, and 10 from the SWIR. The top 20 VIP for the presymptomatic disease time model included eight wavelengths from the SWIR, five from the NIR, and seven from the VIS. The top 20 VIP for the postsymptomatic disease time model included nine wavelengths in the SWIR, six from the NIR, and five from the VIS (Table 2). The improved performance of FOD over untransformed spectra in the $\mathrm{RF}$ model is likely a consequence of its sensitivity to changes in reflectance between adjacent bands rather than magnitude of change at individual wavelengths.

Despite some variation, both PLS-DA and RF identified similar spectral features for discrimination during all disease time points (Fig. 6A) and within the various disease time stages (Supplementary Fig. S4). We should note that the RF classification used wavelengths to binned $10-\mathrm{nm}$ intervals due to the inability of RF to handle large numbers of independent variables compared with PLS-DA; thus, each identified VIP in RF can be considered to account for the influence of nearby bands that might have had high absolute-value standardized coefficients in PLS-DA. The VIP and PLS-DA model standardized coefficients can also be compared with the most correlated NDSIs for disease time stage (Supplementary Fig. S4B, $\mathrm{D}, \mathrm{F}$, and $\mathrm{H}$ ) to corroborate the wavelengths most important for differentiation. NDSI is a univariate method and considers each wavelength independently of all others, which is likely why this method identified differences in the VIS wavelengths as being most important. In contrast, both multivariate methods (PLS-DA and RF) identified important features in the NIR and SWIR for discrimination. There is strong overlap in the spectral features identified by all three analytical methods for each disease development stage (Supplementary Fig. S4) as well as within models fit across all disease time points (Fig. 6).

\section{DISCUSSION}

Using three different discrimination methods (PLS-DA, RF, and NDSI), we found that spectral differences are greatest between US08 and US-23 during early infection ( $24 \mathrm{hpi}$ ). This time period coincided with the greatest difference in $A v r 3 a$ expression between the two clonal lineages. As visualized by the NDSI heatmaps and distribution of most correlated wavelengths, the greatest spectral differences between infections caused by US-08 and US-23 appeared to be during presymptomatic infection stages (early infection and biotrophy). Similarly, PLS-DA cross-validation accuracy was highest during early infection, followed by biotrophy. PLS-DA differentiation (US-08 versus US-23) accuracy was stable across biotrophy, necrotrophy, and sporulation, with accuracy $>91 \%$ and $\kappa=0.82$.

As symptoms developed, differentiation became more difficult and spectral profiles converged. The differences in spectral profiles peaked during the presymptomatic phases of the $P$. infestans disease cycle, particularly during early infection, implying that the underlying processes driving differentiation are greatest during this stage. Five of the six descriptive physiological indices calculated from the growth chamber study showed highly significant differences between US-08 and US-23 during early infection, whereas only two were significantly different during sporulation. The physiological impact of disease on naturally
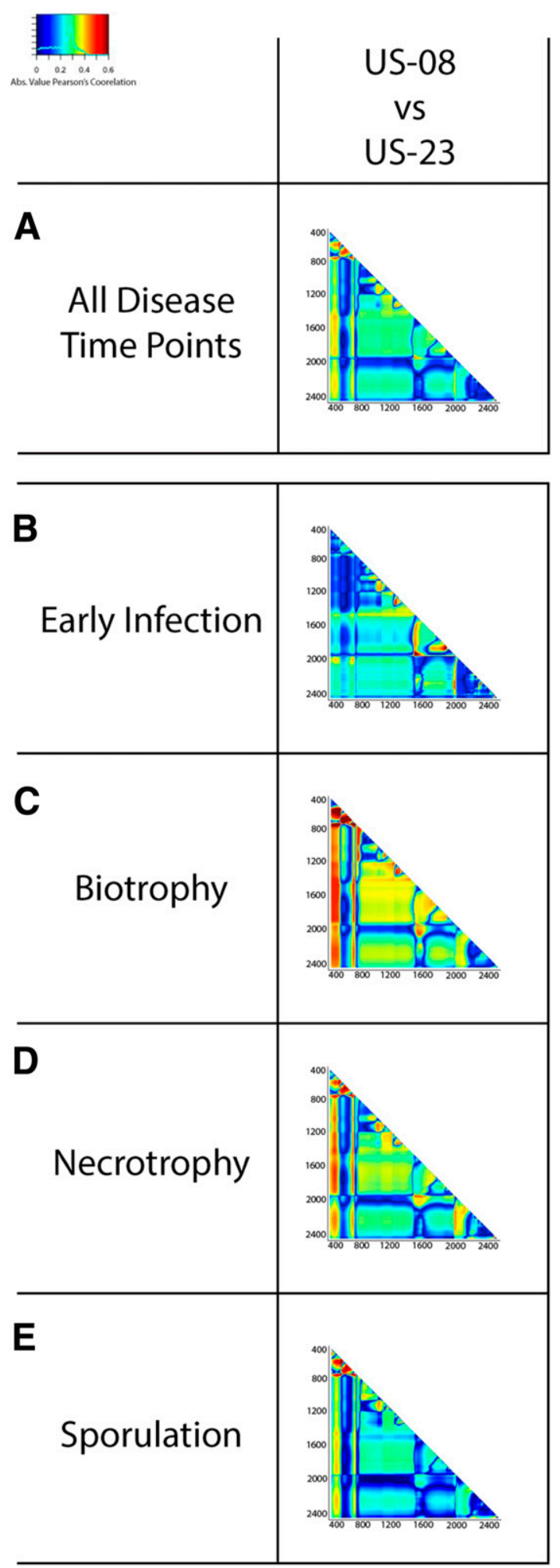

Fig. 5. Normalized differential spectral index (NDSI) heat maps showing wavelength combinations most correlated (absolute value of Pearson's correlation) with a difference between potato leaves inoculated with Phytophthora infestans clonal lineages US-23 and US-08 across disease time. Blue indicates lowest correlation values and, thus, the most similarity between classes at these specified spectral regions, the normalized wavelength combinations that are least changed between US- 23 and US- 08 . Red indicates highest correlation values and, thus, the greatest differences between classes at these specified spectral regions, the normalized wavelength combinations that are most highly changed between US-23 and US-08. Reflectance was calculated from visible to shortwave infrared reflectance ( 400 to $2,500 \mathrm{~nm}$ ) on leaves using a portable spectrometer with contact probe at 12- to 24-h intervals for 5 days. A, All disease time points; $\mathbf{B}$, early infection; $\mathbf{C}$, biotrophy; D, necrotrophy; and $\mathbf{E}$, sporulation. 
inoculated, sporulating samples from commercial production fields was highly similar. Therefore, we conclude that there is greater consistency in plant response to late blight infection as disease progresses, and that the main effects of different clonal lineages are expressed in differing physiological responses early in infection.
Thus, despite the overall similarities between the spectral profiles of US-08- and US-23-infected leaves, we find that clonal lineages can be accurately differentiated across all disease development stages with three approaches. Fine spectral differences exist between foliage of plants infected with highly virulent isolates of

\section{A All Disease Time Points}

PLS-DA Std. Coef. Annotated with RF Three Class Discrimination Top 20 VIP

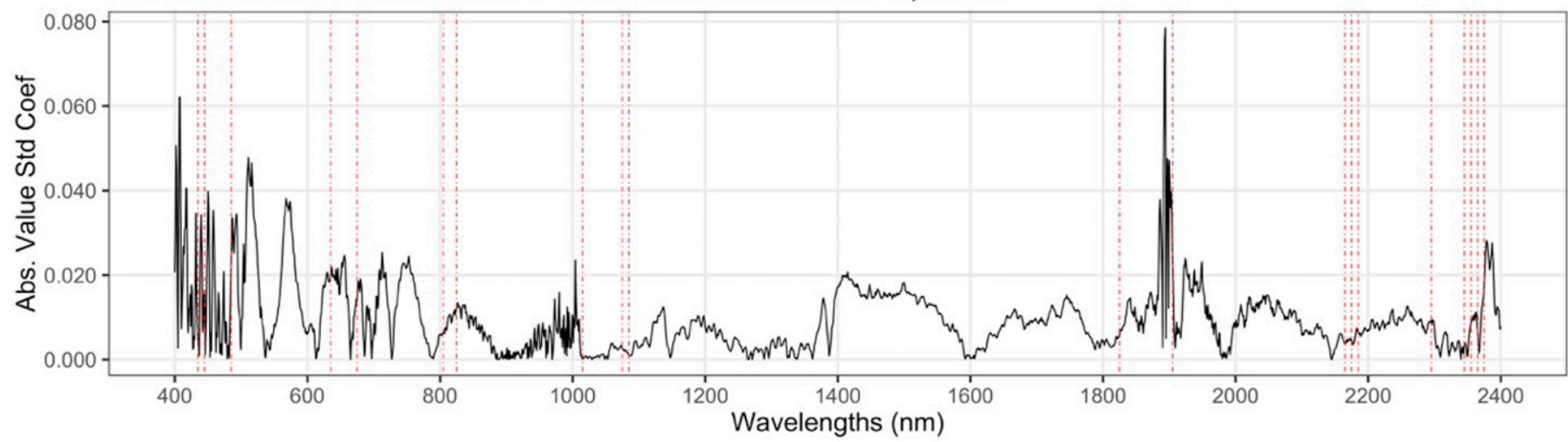

B Distribution of Most Correlated NDSI Wavelengths - US23 vs. US08

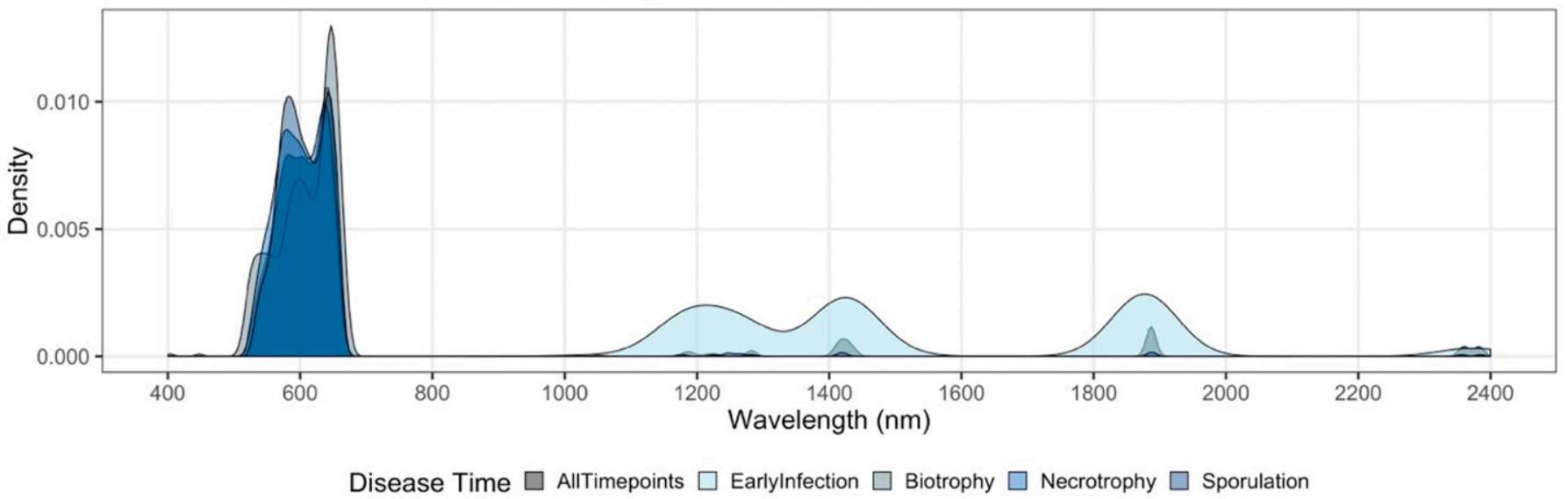

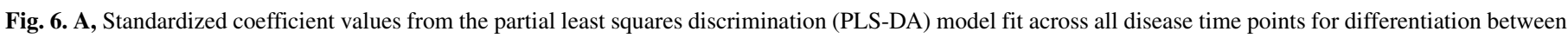

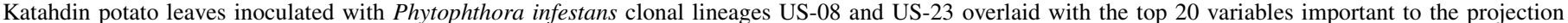

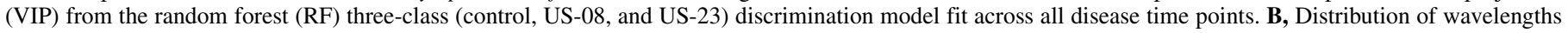

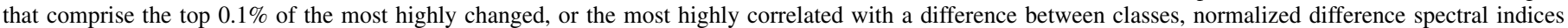
(NDSIs) between potato leaves inoculated with $P$. infestans clonal lineages US-08 and US-23 across disease time.

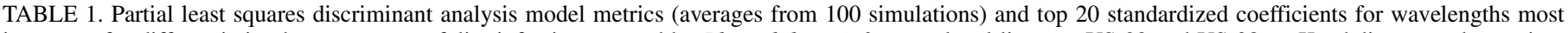

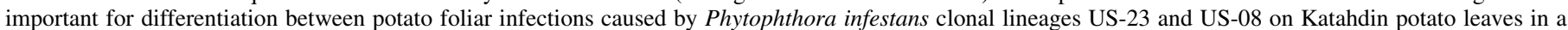

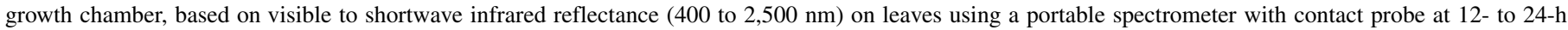
intervals for 5 days

\begin{tabular}{|c|c|c|c|c|c|c|}
\hline \multirow[b]{2}{*}{ Model } & \multicolumn{2}{|l|}{ Trait } & \multicolumn{2}{|c|}{$\begin{array}{l}\text { Internal cross- } \\
\text { validation }\end{array}$} & \multirow[b]{2}{*}{ Components } & \multirow[b]{2}{*}{ Coefficients (nm) } \\
\hline & Accuracy $(\%)$ & $\kappa$ & Accuracy $(\%)$ & $\kappa$ & & \\
\hline All disease time points & 96.04 & 0.92 & 94.98 & 0.90 & 14 & $\begin{array}{r}1895,1894,1893,512,1901,517,1892,511,513,518, \\
514,510,516,515,1902,451,1904,1887,519,1903\end{array}$ \\
\hline Early infection & 99.88 & 1.00 & 98.34 & 0.97 & 12 & $\begin{array}{l}1895,1894,1892,1893,1901,1903,1904,1870,1902, \\
1871,1869,2379,2378,511,1873,517,1905,1868, \\
513,1872\end{array}$ \\
\hline Biotrophy & 97.63 & 0.95 & 95.14 & 0.90 & 9 & $\begin{array}{l}1895,1894,1893,1896,1892,1888,1887,1889,1890, \\
\quad 1886,1883,657,658,1880,1882,1885,1879,1881, \\
659,661\end{array}$ \\
\hline Necrotrophy & 93.35 & 0.87 & 90.30 & 0.81 & 7 & $\begin{array}{l}1899,1896,1898,1894,1895,1897,1893,1901,1887, \\
2380,2381,2379,1902,2378,2382,2323,1886,1888, \\
2377,2384\end{array}$ \\
\hline Sporulation & 92.82 & 0.85 & 88.88 & 0.77 & 7 & $\begin{array}{l}1899,1897,1898,1893,1894,1895,1896,1892,2380, \\
2381,2379,2382,2378,2383,2377,2376,2384,2375, \\
2385,2386\end{array}$ \\
\hline
\end{tabular}


two clonal lineages. The underlying biochemical and physiological processes driving differentiation appear to be fairly consistent across disease stages but strongest and easiest to sense during presymptomatic infection. Quantification of this phenomenon is made possible by the use of VSWIR hyperspectral data, in which hundreds to thousands of wavelengths are measured, some of which show common responses to infestation between lineages, thus enabling discrimination between healthy and diseased (Gold et al. 2019; Gold et al. in press), whereas others differ, enabling detection of minute differences. With further study and independent validation, these differences could be harnessed for in-field, rapid clonal lineage identification. To move toward this goal, a greater number of isolates and clonal lineages must be tested to confirm these initial findings. Despite the need for further validation, this work establishes proof of concept and supports the future use of in-situ spectroscopy as a basic tool for strain and pathovar differentiation.

To the best of our knowledge, this is the first study demonstrating that in-situ or contact spectroscopy can differentiate between plant infections caused by different variants of pathogens, or pathovars. The capacity to distinguish between potato late blight clonal lineages with sporangial-level analysis has been previously established with a related technique, Fourier transformed infrared (FTIR) microspectroscopy (Pomerantz et al. 2014). These authors established that it is possible to use spectroscopic methodology to quantify the sum total of physiological and biochemical differences in spores between pathovars that result in different light reflectance. Contact vegetative spectroscopy differs from FTIR spectroscopy because it is less invasive, nondestructive, faster, covers a reduced wavelength range (400 to $2,500 \mathrm{~nm}$ instead of 400 to $4,000 \mathrm{~nm}$ ), does not require a laboratory set up, and is based on measurements made on vegetative tissue. Our work here builds upon these findings by expanding the known capacity for spectroscopic methods to differentiate between pathovars to a method more suited to rapid and in-field assessment. As the discipline moves toward pathovar identification with spectroscopic methods, a crucial next step will be independent validation and classification from both this study and Pomerantz et al. (2014).

Varying isolate aggressiveness may be responsible for spectral differences seen in the VIS wavelengths across all disease stages. Alisaac et al. (2018) proposed that the differences seen between the two Fusarium spp. in their study were due to isolate virulence rather than a true difference in symptomology. Potato is the preferred host for US-08, whereas US-23 has equal preference for potato and tomato (Halterman and Gevens 2013). This may contribute to contrasts in aggressiveness, disease progression, and the resulting intensity of spectral differences between leaves infected by the two lineages. We recognize that potential differences in isolate aggressiveness can also be due to culture age (Fry et al. 2019) or environment. We attempted to reduce variation due to these confounding factors by comparing between two highly virulent isolates collected during the same field season from the same host plant. Future work must include a larger collection of isolates and lineages to better understand differences that may be associated with specific clonal lineages, rather than due to variation within a clonal lineage. This is a critical next step because, although all individuals of a clonal lineage are descended from a single genotype, there can be diversity within that population of individuals (Fry and Goodwin 1997; Seidl and Gevens 2013; Seidl Johnson et al. 2014, 2015a). These further investigations will validate the proof-of-concept work presented here.

Once $P$. infestans has established haustorial connections with its host, it is able to secrete effectors into host cells to modulate host plant processes (Fry 2008). These effectors have diverse roles throughout the infection process, from silencing host defense pathways to inducing cell death. In the United States, there has been little evidence of sexual recombination in P. infestans leading to persistent, highly infectious genotypes. Rather, we see emergences and disappearances of dominant, asexually derived clonal lineages with different levels of virulence, host preferences, and mefenoxam sensitivity. It stands to reason that diverse populations of clonal lineages in the United States will have different levels of effector expression and subsequent impact on host processes. In this study, we assessed the expression of two RXLR effectors ( $A v r 3 a$ and SFI3) in order to relate underlying pathogen biology and disease physiology to differences in spectral reflectance between US-08 and US-23. Silencing work has shown that Avr3a is essential for infection establishment, and that its expression is upregulated throughout the entire infection cycle, beginning with a $>100$-fold increase in germinating cysts undergoing appressorium development and peaking during the biotrophic phase of infection (Armstrong et al. 2005; Zheng et al. 2014). SFI3, an RXLR effector and known suppressor of early Flg22-induced immune response, is also upregulated during the biotrophic phase of infection (Zheng et al. 2014). Our work suggests that differences in effector expression, and likely the downstream impact of effector expression on plant biochemistry, physiology, and morphology, may contribute to spectral differences between clonal lineages during early infection stages.

SWIR wavelengths are important for discrimination between infections caused by closely related species (Baranowski et al. 2015). We find this to be true for differentiation between clonal

TABLE 2. Random forest model results for discrimination between control and Phytophthora infestans clonal lineages US-23 and US-08 on Katahdin potato leaves in a growth chamber, based on visible to shortwave infrared reflectance (400 to 2,500 nm) on leaves using a portable spectrometer with contact probe ${ }^{\mathrm{a}}$

\begin{tabular}{|c|c|c|c|c|}
\hline \multirow[b]{2}{*}{ Model } & \multicolumn{4}{|r|}{ Multiclass discrimination } \\
\hline & Accuracy $(\%)$ & $\kappa$ & mtry & Top 20 VIP $(\mathrm{nm})$ \\
\hline \multicolumn{5}{|l|}{ Primary } \\
\hline Presymptomatic & 59.82 & 0.38 & 36 & $\begin{array}{l}1405,1375,1395,715,1385,725,415,1365,735,675,1415,1425,1345,425 \\
\quad 1895,2375,485,665,705,1355\end{array}$ \\
\hline Postsymptomatic & 60.74 & 0.41 & 16 & $\begin{array}{l}455,705,545,465,565,555,475,715,665,485,445,675,685,655,535,575, \\
\quad 435,2375,415,1375\end{array}$ \\
\hline All disease time & 69.03 & 0.49 & 21 & $\begin{array}{l}2375,1825,2165,2355,635,1075,2365,445,675,805,435,485,2295,1015 \\
\quad 2175,1905,1085,825,2185,2345\end{array}$ \\
\hline Presymptomatic & 75.89 & 0.63 & 1 & $\begin{array}{l}515,1165,635,2365,1785,2175,645,535,2155,755,1195,605,1175,775 \\
\quad 1795,705,1865,1905,2385,575\end{array}$ \\
\hline Postsymptomatic & 67.89 & 0.43 & 6 & $\begin{array}{l}2375,675,825,1825,2345,1905,2165,805,815,2355,2365,1265,835,545, \\
\quad 515,535,1075,595,1015,1255\end{array}$ \\
\hline
\end{tabular}

\footnotetext{
a To achieve an appropriate sample number, disease time stages were binned according to pre- or postsymptom development. Accuracy and $\kappa$ indicate average classification accuracy and $\kappa$, respectively, across 10 model simulations; $m$ try $=$ the optimal number of randomly selected predictors chosen at each tree branch chosen for the specified model; and VIP = the top 20 variables important to the projection across all 10 model simulations.
} 
lineages as well. Differences in SWIR wavelengths between lineages likely result from differences in underlying biochemistry and physiology. SWIR wavelengths correspond to a range of physiological and chemical characteristics, including nitrogen bonds, structural carbon (such as lignin and cellulose) and leaf water content (Curran 1989). RF discrimination identified the $1,825-\mathrm{nm}$ region of wavelengths to be in the top $20 \mathrm{VIP}$ in all three models, a region that strongly corresponds to cellulose. Cellulose is an essential component of plant cell walls and is compromised by late blight infection, particularly during necrotrophy (Erwin and Ribeiro 1996). Wavelengths between 2,350 and 2,400 nm, a region known to correspond to cellulose structure and concentration (Curran 1989), were also important for PLS-DA differentiation. These wavelengths were also noted to be of importance for late blight differentiation from healthy leaves (Gold et al. 2019). The importance of these spectral regions suggests that leaf structure is compromised differentially by the two lineages, in terms of either rate of decline or severity of decline, which can be related to clonal lineage or isolate virulence. Cellulose also relates to LMA which, in our study, was significantly affected by the interaction of disease time and treatment. Changes in LMA can be related to the trade-off between investment in longevity versus growth in leaves (Poorter et al. 2009). When LMA is lower, plants are investing resources in processes other than those related to structure building (e.g., cellulose) such as rapid photosynthesis during periods of calm or defense during periods of stress (Poorter et al. 2009).

Total phenolics concentration was found to be significantly different between US-08 and US-23 P. infestans infections and across all treatments at all disease stages, and between diseased and nondiseased field-collected leaves. This suggests that leaf phenolic production is differentially affected by respective clonal lineage infections. Secondary metabolites with phenolic structures such as salicylic acid are well known to be important for plant defense (Nicholson and Hammerschmidt 1992). Moreover, the $P A L-1$ gene encodes for a lyase that controls the production of phenolic compounds, and has been shown to be differentially expressed during infection by different $P$. infestans clonal lineages (Wang et al. 2008). Secondary metabolites are differentially produced during the early stages of tomato late blight infection (Galeano Garcia et al. 2018). Previous work has shown that it is possible to use spectroscopy to quantify defense-associated compounds (Couture et al. 2013, 2016; Herrmann et al. 2020; Kokaly and Skidmore 2015). Specific spectral quantification of differentially expressed phenolic and defense compounds may one day allow for more accurate and robust characterization of different clonal lineages in field settings, especially as symptoms are more manifest.

Relative proportions of foliar sugars and starches may contribute to the spectral differences between US-08 and US-23 during presymptomatic infection. We found that leaf starch and sugar concentration differed between US-08- and US-23-infected leaves during presymptomatic disease stages, and that wavelengths associated with these compounds were found in the top 20 VIP in all three RF models. The allozyme assay currently used for clonal lineage characterization identifies different isozymes of the glucose-6-phosphate isomerase enzyme. This enzyme plays a key role in both glycolysis and gluconeogenesis and is known to vary between US-08 and US-23 (Hu et al. 2012). Both sugars and starch are products of these pathways. Differential allocation in metabolic pathways between the lineages could be related to underlying differences in the function of this enzyme. More experimentation is needed to determine whether these observed differences are due to increased pathogen consumption or reduced plant production. Regardless of origin, these differences are a potential direction to pursue for more accurate differentiation.

Wavelengths in the VIS range (500 to $750 \mathrm{~nm}$ ) constituted a small portion of the wavelengths needed for postsymptomatic, multivariate discrimination despite being important for univariate NDSI discrimination. This work suggests that postsymptomatic differentiation at more advanced and analytically difficult deployment levels such as aircraft or satellite may not be possible without SWIR wavelengths. Differences in VIS wavelengths often correspond to changes in chlorophyll concentration (Curran 1989) and can be strongly affected by many abiotic or biotic stresses (Lichtenthaler et al. 1998; Mahlein 2016; Mahlein et al. 2018). A robust study across multiple pathogen isolates and clonal lineages to determine field differentiation capacity with and without SWIR wavelengths would greatly support, and further validate, our proofof-concept work.

Discerning minor differences between pathotypes or genotypes of a plant pathogen with rapid, nondestructive, and noninvasive sensing offers a high-value tool for advancing research discoveries in disease dispersal and detection, as well as for advancing robust pathogen detection capacity in seed potato production or commercial fields for more rapid and effective response. We found that the underlying biochemical and physiological processes affected by $P$. infestans infection that result in changes in the NIR and SWIR are similar between representative isolates of the US-08 and US-23 clonal lineages at symptomatic stages but were more different during presymptomatic infection phases. This finding should guide further exploration of hyperspectral systems application for field usage, especially for early detection. Finally, the effect of cultivar on diseased spectral profile must be taken into consideration, because different cultivars appear to react differently to early stage $P$. infestans infection (Gold et al. 2019).

\section{ACKNOWLEDGMENTS}

We thank J. Hammel, M. Chen, S. Jordan, A. Mazur, E. H. Wagner, J. Spychalla, H. Knight, and T. Wu for their assistance and support in data collection; and our peer reviewers and senior editor for their helpful suggestions and thoughtful constructive criticism.

\section{LITERATURE CITED}

Alisaac, E., Behmann, J., Kuska, M. T., Dehne, H.-W., and Mahlein, A.-K. 2018. Hyperspectral quantification of wheat resistance to Fusarium head blight: Comparison of two Fusarium species. Eur. J. Plant Pathol. 152: 869-884.

Anderson, J. B., and Kohn, L. M. 1995. Clonality in soilborne, plantpathogenic fungi. Annu. Rev. Phytopathol. 33:369-391.

Anderson, M. J., and Walsh, D. C. I. 2013. PERMANOVA, ANOSIM, and the Mantel test in the face of heterogeneous dispersions: What null hypothesis are you testing? Ecol. Monogr. 83:557-574.

Arens, N., Backhaus, A., Döll, S., Fischer, S., Seiffert, U., and Mock, H.-P. 2016. Non-invasive presymptomatic detection of Cercospora beticola infection and identification of early metabolic responses in sugar beet. Front. Plant Sci. 7:1377.

Armstrong, M. R., Whisson, S. C., Pritchard, L., Bos, J. I. B., Venter, E., Avrova, A. O., Rehmany, A. P., Böhme, U., Brooks, K., Cherevach, I., Hamlin, N., White, B., Fraser, A., Lord, A., Quail, M. A., Churcher, C., Hall, N., Berriman, M., Huang, S., Kamoun, S., Beynon, J. L., and Birch, P. R. J. 2005. An ancestral oomycete locus contains late blight avirulence gene Avr3a, encoding a protein that is recognized in the host cytoplasm. Proc. Natl. Acad. Sci. U.S.A. 102:7766-7771.

Asner, G. P., and Martin, R. E. 2008. Spectral and chemical analysis of tropical forests: Scaling from leaf to canopy levels. Remote Sens. Environ. 112:3958-3970.

Asner, G. P., and Martin, R. E. 2011. Canopy phylogenetic, chemical and spectral assembly in a lowland Amazonian forest. New Phytol. 189:999-1012.

Baranowski, P., Jedryczka, M., Mazurek, W., Babula-Skowronska, D., Siedliska, A., and Kaczmarek, J. 2015. Hyperspectral and thermal imaging of oilseed rape (Brassica napus) response to fungal species of the genus Alternaria. PLoS One 10:e0122913.

Barratt, R., and Horsfall, J. 1945. An improved grading system for measuring plant disease. Phytopathology 35:655.

Bergsträsser, S., Fanourakis, D., Schmittgen, S., Cendrero-Mateo, M. P., Jansen, M., Scharr, H., and Rascher, U. 2015. HyperART: Non-invasive quantification of leaf traits using hyperspectral absorption-reflectancetransmittance imaging. Plant Methods 11:1.

Breiman, L. 2001. Random forests. Mach. Learn. 45:5-32.

Cohen, J. 1960. A coefficient of agreement for nominal scales. Educ. Psychol. Meas. 20:37-46. 
Couture, J. J., Serbin, S. P., and Townsend, P. A. 2013. Spectroscopic sensitivity of real-time, rapidly induced phytochemical change in response to damage. New Phytol. 198:311-319.

Couture, J. J., Singh, A., Charkowski, A. O., Groves, R. L., Gray, S. M., Bethke, P. C., and Townsend, P. A. 2018. Integrating spectroscopy with potato disease management. Plant Dis. 102:2233-2240.

Couture, J. J., Singh, A., Rubert-Nason, K. F., Serbin, S. P., Lindroth, R. L., and Townsend, P. A. 2016. Spectroscopic determination of ecologically relevant plant secondary metabolites. Methods Ecol. Evol. 7:1402-1412.

Curran, P. J. 1989. Remote sensing of foliar chemistry. Remote Sens. Environ. 30:271-278.

Danies, G., Small, I. M., Myers, K., Childers, R., and Fry, W. E. 2013. Phenotypic characterization of recent clonal lineages of Phytophthora infestans in the United States. Plant Dis. 97:873-881.

Deahl, K. L., Goth, R. W., Young, R., Sinden, S. L., and Gallegly, M. E. 1991. Occurrence of the A2 mating type of Phytophthora infestans in potato fields in the United States and Canada. Am. Potato J. 68:717-725.

Deahl, K. L., Inglis, D. A., and DeMuth, S. P. 1993. Testing for resistance to metalaxyl in Phytophthora infestans isolates from northwestern Washington. Am. Potato J. 70:779-795.

Demetriades-Shah, T. H., Steven, M. D., and Clark, J. A. 1990. High resolution derivative spectra in remote sensing. Remote Sens. Environ. 33:55-64.

Erwin, D. C., and Ribeiro, O. K. 1996. Phytophthora Diseases Worldwide. American Phytopathological Society, St. Paul, MN, U.S.A.

Frost, K. E., Seidel Johnson, A. C., and Gevens, A. J. 2015. Survival of isolates of the US-22, US-23, and US-24 clonal lineages of Phytophthora infestans by asexual means in tomato seeds at cold temperatures. Plant Dis. 100: $180-187$.

Fry, W. 2008. Phytophthora infestans: The plant (and R gene) destroyer. Mol. Plant Pathol. 9:385-402.

Fry, W. E., Birch, P. R. J., Judelson, H. S., Grünwald, N. J., Danies, G., Everts, K. L., Gevens, A. J., Gugino, B. K., Johnson, D. A., Johnson, S. B., McGrath, M. T., Myers, K, L., Ristaino, J. B., Roberts, P. D., Secor, G., and Smart, C. D. 2015. Five reasons to consider Phytophthora infestans a reemerging pathogen. Phytopathology 105:966-981.

Fry, W. E., and Goodwin, S. B. 1997. Re-emergence of potato and tomato late blight in the United States. Plant Dis. 81:1349-1357.

Fry, W. E., McGrath, M. T., Seaman, A., Zitter, T. A., McLeod, A., Danies, G., Small, I. M., Myers, K., Everts, K., Gevens, A. J., Gugino, B. K., Johnson, S. B., Judelson, H., Ristaino, J., Roberts, P., Secor, G., Seebold, K., Jr., Snover-Clift, K., Wyenandt, A., Grünwald, N. J., and Smart, C. D. 2013. The 2009 late blight pandemic in the eastern United States-Causes and results. Plant Dis. 97:296-306.

Fry, W. E., Patev, S. P., Myers, K. L., Bao, K., and Fei, Z. 2019. Phytophthora infestans sporangia produced in culture and on tomato leaflet lesions show marked differences in indirect germination rates, aggressiveness, and global transcription profiles. Mol. Plant-Microbe Interact. 32:515-526.

Galeano Garcia, P., Neves dos Santos, F., Zanotta, S., Eberlin, M. N., and Carazzone, C. 2018. Metabolomics of Solanum lycopersicum infected with Phytophthora infestans leads to early detection of late blight in asymptomatic plants. Molecules 23:3330.

Gao, B. 1996. NDWI-A normalized difference water index for remote sensing of vegetation liquid water from space. Remote Sens. Environ. 58: 257-266.

Gillon, D., Houssard, C., and Joffre, R. 1999. Using near-infrared reflectance spectroscopy to predict carbon, nitrogen and phosphorus content in heterogeneous plant material. Oecologia 118:173-182.

Gold, K. M., Gevens, A. J., and Townsend, P. A. 2019. System for Detection of Disease In Plants. U.S. Patent App. 16/251,415.

Gold, K. M., Townsend, P. A., Herrmann, I., and Gevens, A. J. 2019. Investigating potato late blight physiological differences across potato cultivars with spectroscopy and machine learning. Plant Science. https://doi.org/ 10.1016/j.plantsci.2019.110316

Goodwin, S. B., Schneider, R. E., and Fry, W. E. 1995. Use of cellulose-acetate electrophoresis for rapid identification of allozyme genotypes of Phytophthora infestans. Plant Dis. 79:1181-1185.

Halterman, D., and Gevens, A. J. 2013. Phytophthora infestans in the United States. Pages 68-78 in: Phytophthora: A Global Perspective. K. Lamour, ed. CABI Plant Protection Series. CAB International.

Hansen, Z. R., Small, I. M., Mutschler, M., Fry, W. E., and Smart, C. D. 2014. Differential susceptibility of 39 tomato varieties to Phytophthora infestans clonal lineage US-23. Plant Dis. 98:1666-1670.

Hastie, T., Tibshirani, R., and Friedman, J. 2009. The Elements of Statistical Learning: Data Mining, Inference, and Prediction. Springer Series in Statistics. Springer New York, New York.

Heim, R. H. J., Wright, I. J., Chang, H.-C., Carnegie, A. J., Pegg, G. S., Lancaster, E. K., Falster, D. S., and Oldeland, J. 2018. Detecting myrtle rust (Austropuccinia psidii) on lemon myrtle trees using spectral signatures and machine learning. Plant Pathol. 67:1114-1121.
Henriquez, M. A., Adam, L. R., and Daayf, F. 2012. Alteration of secondary metabolites' profiles in potato leaves in response to weakly and highly aggressive isolates of Phytophthora infestans. Plant Physiol. Biochem. 57: 8-14

Herrmann, I., Bdolach, E., Montekyo, Y., Rachmilevitch, S., Townsend, P. A., and Karnieli, A. 2020. Assessment of maize yield and phenology by dronemounted superspectral camera. Precis. Agric. 21:51-76.

Herrmann, I., Vosberg, S. K., Ravindran, P., Singh, A., Chang, H.-X., Chilvers, M. I., Conley, S. P., and Townsend, P. A. 2018. Leaf and canopy level detection of Fusarium virguliforme (sudden death syndrome) in soybean. Remote Sens. 10:426.

Hu, C.-H., Perez, F. G., Donahoo, R., McLeod, A., Myers, K., Ivors, K., Secor, G., Roberts, P. D., Deahl, K. L., Fry, W. E., and Ristino, J. B. 2012. Recent genotypes of Phytophthora infestans in the eastern United States reveal clonal populations and reappearance of mefenoxam sensitivity. Plant Dis. 96:1323-1330.

Immitzer, M., Atzberger, C., and Koukal, T. 2012. Tree species classification with random forest using very high spatial resolution 8-band worldview-2 satellite data. Remote Sens. 4:2661-2693.

Kokaly, R. F., Asner, G. P., Ollinger, S. V., Martin, M. E., and Wessman, C. A. 2009. Characterizing canopy biochemistry from imaging spectroscopy and its application to ecosystem studies. Remote Sens. Environ. 113: S78-S91.

Kokaly, R. F., and Skidmore, A. K. 2015. Plant phenolics and absorption features in vegetation reflectance spectra near 1.66 $\mu \mathrm{m}$. Int. J. Appl. Earth Obs. Geoinf. 43:55-83.

Kuhn, M. 2015. caret: Classification and Regression Training. https://rdrr.io/ cran/caret/

Kuska, M., Wahabzada, M., Leucker, M., Dehne, H.-W., Kersting, K., Oerke, E.-C., Steiner, U., and Mahlein, A.-K. 2015. Hyperspectral phenotyping on the microscopic scale: Towards automated characterization of plantpathogen interactions. Plant Methods 11:28.

Lamour, K. H., and Hausbeck, M. K. 2002. The spatiotemporal genetic structure of Phytophthora capsici in Michigan and implications for disease management. Phytopathology 92:681-684.

Landis, J. R., and Koch, G. G. 1977. The measurement of observer agreement for categorical data. Biometrics 33:159-174.

Lenth, R. 2018. Emmeans: Estimated marginal means, aka least-squares means. R Package Version 1. https://cran.r-project.org/web/packages/emmeans/ index.html

Lichtenthaler, H. K., Wenzel, O., Buschmann, C., and Gitelson, A. 1998. Plant stress detection by reflectance and fluorescence. Ann. N. Y. Acad. Sci. 851: 271-285.

Liu, Z.-Y., Wu, H.-F., and Huang, J.-F. 2010. Application of neural networks to discriminate fungal infection levels in rice panicles using hyperspectral reflectance and principal components analysis. Comput. Electron. Agric. 72:99-106.

Mahlein, A.-K. 2016. Plant disease detection by imaging sensors-Parallels and specific demands for precision agriculture and plant phenotyping. Plant Dis. 100:241-251.

Mahlein, A.-K., Kuska, M. T., Behmann, J., Polder, G., and Walter, A. 2018. Hyperspectral sensors and imaging technologies in phytopathology: State of the art. Annu. Rev. Phytopathol. 56:535-558.

Mahlein, A.-K., Rumpf, T., Welke, P., Dehne, H.-W., Plümer, L., Steiner, U., and Oerke, E.-C. 2013. Development of spectral indices for detecting and identifying plant diseases. Remote Sens. Environ. 128:21-30.

Mahlein, A.-K., Steiner, U., Dehne, H.-W., and Oerke, E.-C. 2010. Spectral signatures of sugar beet leaves for the detection and differentiation of diseases. Precis. Agric. 11:413-431.

Mahlein, A.-K., Steiner, U., Hillnhütter, C., Dehne, H.-W., and Oerke, E.-C. 2012. Hyperspectral imaging for small-scale analysis of symptoms caused by different sugar beet diseases. Plant Methods 8:3.

Mevik, B.-H., Wehrens, R., and Liland, K. H. 2011. pls: Partial least squares and principal component regression. R Package Version 2. https://cran.rproject.org/web/packages/pls/index.html

Nicholson, R. L., and Hammerschmidt, R. 1992. Phenolic compounds and their role in disease Resistance. Annu. Rev. Phytopathol. 30:369-389.

Oksanen, J., Blanchet, F. G., Kindt, R., Legendre, P., Minchin, P. R., O’Hara, R., Simpson, G. L., Solymos, P., Henry, M., Stevens, H., Szoecs, E., and Wagner, H. 2013. Package 'vegan.' Community Ecology Package. Version 2. https://cran.r-project.org/web/packages/vegan/index.html

Pariaud, B., Ravigné, V., Halkett, F., Goyeau, H., Carlier, J., and Lannou, C. 2009. Aggressiveness and its role in the adaptation of plant pathogens. Plant Pathol. 58:409-424.

Petisco, C., García-Criado, B., Mediavilla, S., Vázquez de Aldana, B. R., Zabalgogeazcoa, I., and García-Ciudad, A. 2006. Near-infrared reflectance spectroscopy as a fast and non-destructive tool to predict foliar organic constituents of several woody species. Anal. Bioanal. Chem. 386: 1823-1833. 
Pinheiro, J., Bates, D., DebRoy, S., Sarkar, D., and R Core Team. 2013. nlme: Linear and nonlinear mixed effects models. R Package Version 3.1-111. https://cran.r-project.org/web/packages/nlme/index.html

Pomerantz, A., Cohen, Y., Shufan, E., Ben-Naim, Y., Mordechai, S., Salman, A., and Huleihel, M. 2014. Characterization of Phytophthora infestans resistance to mefenoxam using FTIR spectroscopy. J. Photochem. Photobiol. B 141:308-314.

Poorter, H., Niinemets, Ü., Poorter, L., Wright, I. J., and Villar, R. 2009. Causes and consequences of variation in leaf mass per area (LMA): A metaanalysis. New Phytol. 182:565-588.

Reich, P. B. 2014. The world-wide 'fast-slow' plant economics spectrum: A traits manifesto. J. Ecol. 102:275-301.

Rumpf, T., Mahlein, A.-K., Steiner, U., Oerke, E.-C., Dehne, H.-W., and Plümer, L. 2010. Early detection and classification of plant diseases with support vector machines based on hyperspectral reflectance. Comput. Electron. Agric. 74:91-99.

Seidl, A. C., and Gevens, A. J. 2013. Characterization and distribution of three new clonal lineages of Phytophthora infestans causing late blight in Wisconsin from 2009 to 2012. Am. J. Potato Res. 90:551-560.

Seidl Johnson, A. C., and Gevens, A. J. 2014. Investigating the host range of the US-22, US-23, and US-24 clonal lineages of Phytophthora infestans on solanaceous cultivated plants and weeds. Plant Dis. 98:754-760.

Seidl Johnson, A. C., Frost, K. E., Rouse, D. I., and Gevens, A. J. 2015a. Effect of temperature on growth and sporulation of US-22, US-23, and US-24 clonal lineages of Phytophthora infestans and implications for late blight epidemiology. Phytopathology 105:449-459.

Seidl Johnson, A. C., Jordan, S. A., and Gevens, A. J. 2015b. Efficacy of organic and conventional fungicides and impact of application timing on control of tomato late blight caused by US-22, US-23, and US-24 isolates of Phytophthora infestans. Plant Dis. 99:641-647.

Serbin, S. P. 2012. Spectroscopic determination of leaf nutritional, morphological, and metabolic traits. Ph.D. thesis. https://ui.adsabs.harvard.edu/abs/ 2012PhDT.......189S/abstract

Singh, A., Serbin, S. P., McNeil, B. E., Kingdon, C. C., and Townsend, P. A. 2015. Imaging spectroscopy algorithms for mapping canopy foliar chemical and morphological traits and their uncertainties. Ecol. Appl. 25:2180-2197.

Small, I., Myers, K., Danies, G., Roy, S. G., Bekoscke, K., and Fry, W. 2012. Characterization of recent clonal lineages of Phytophthora infestans in the United States using microsatellite markers. (Abstr.) Phytopathology 102:110.

Stevens, A., and Ramirez-Lopez, L. 2014. An introduction to the prospectr package. R Package Vignette. https://cran.r-project.org/web/packages/prospectr/ vignettes/prospectr-intro.pdf
Thomas, S., Behmann, J., Steier, A., Kraska, T., Muller, O., Rascher, U., and Mahlein, A.-K. 2018. Quantitative assessment of disease severity and rating of barley cultivars based on hyperspectral imaging in a non-invasive, automated phenotyping platform. Plant Methods 14:45.

Wahabzada, M., Mahlein, A.-K., Bauckhage, C., Steiner, U., Oerke, E.-C., and Kersting, K. 2015. Metro maps of plant disease dynamics-Automated mining of differences using hyperspectral images. PLoS One 10:e0116902.

Wang, X., Hadrami, A. El, Adam, L. R., and Daayf, F. 2008. Differential activation and suppression of potato defence responses by Phytophthora infestans isolates representing US-1 and US-8 genotypes. Plant Pathol. 57: 1026-1037.

Wold, S., Sjöström, M., and Eriksson, L. 2001. PLS-regression: A basic tool of chemometrics. Chemom. Intell. Lab. Syst. 58:109-130.

Worley, B., and Powers, R. 2013. Multivariate analysis in metabolomics. Curr. Metabolomics 1:92-107.

Wright, I. J., Reich, P. B., Westoby, M., Ackerly, D. D., Baruch, Z., Bongers, F., Cavender-Bares, J., Chapin, T., Cornelissen, H. C., Diemer, M., Flexas, J., Garnier, E., Groom, P. K., Gulias, J., Hikosaka, K., Lamont, B. B., Lee, T., Lee, W., Lusk, C., Midgley, J. J., Navas, M.-L., Niinemets, Ü., Oleksyn, J., Osada, N., Poorter, H., Poot, P., Prior, L., Pyankov, V. I., Roumet, C., Thomas, S. C., Tjoelker, M. G., Veneklaas, E. J., and Villar, R. 2004. The worldwide leaf economics spectrum. Nature 428:821-827.

Yuan, M., Couture, J. J., Townsend, P. A., Ruark, M. D., and Bland, W. L. 2016. Spectroscopic determination of leaf nitrogen concentration and mass per area in sweet corn and snap bean. Agron. J. 108:2519-2526.

Zarco-Tejada, P. J., Camino, C., Beck, P. S. A., Calderon, R., Hornero, A., Hernández-Clemente, R., Kattenborn, T., Montes-Borrego, M., Susca, L., Morelli, M., Gonzalez-Dugo, V., North, P. R. J., Landa, B. B., Boscia, D., Saponari, M., and Navas-Cortes, J. A. 2018. Previsual symptoms of Xylella fastidiosa infection revealed in spectral plant-trait alterations. Nat. Plants 4 : 432-439.

Zhai, Y., Cui, L., Zhou, X., Gao, Y., Fei, T., and Gao, W. 2013. Estimation of nitrogen, phosphorus, and potassium contents in the leaves of different plants using laboratory-based visible and near-infrared reflectance spectroscopy: Comparison of partial least-square regression and support vector machine regression methods. Int. J. Remote Sens. 34:2502-2518.

Zheng, X., McLellan, H., Fraiture, M., Liu, X., Boevink, P. C., Gilroy, E. M., Chen, Y., Kandel, K., Sessa, G., Birch, P. R. J., and Brunner, F. 2014. Functionally redundant RXLR effectors from Phytophthora infestans act at different steps to suppress early flg22-triggered immunity. PLoS Pathog. 10: e1004057. 